\title{
RANDOMIZED ALGORITHMS FOR HIGH QUALITY TREATMENT PLANNING IN VOLUMETRIC MODULATED ARC THERAPY
}

\author{
YU YANG*, BIN DONG ${ }^{\dagger}$, AND ZAIWEN WEN ${ }^{\ddagger}$
}

\begin{abstract}
In recent years, volumetric modulated arc therapy (VMAT) has been becoming a more and more important radiation technique widely used in clinical application for cancer treatment. One of the key problems in VMAT is treatment plan optimization, which is complicated due to the constraints imposed by the equipments involved. In this paper, we consider a model with four major constraints: the bound on the beam intensity, an upper bound on the rate of the change of the beam intensity, limit on the moving speed of leaves of the multi-leaf collimator (MLC) and its directional-convexity. We solve the model by a two-stage algorithm: performing minimization with respect to the shapes of the aperture and the beam intensities alternatively. Specifically, the shapes of the aperture are obtained by a greedy algorithm whose performance is enhanced by random sampling in the leaf pairs with a decremental rate. The beam intensity is optimized using a gradient projection method with nonmonotonic line search. We further improve the proposed algorithm by an incremental random importance sampling of the voxels to reduce the computational cost of the evaluation of the energy function. Numerical simulations on two clinical data sets demonstrate that our method is highly competitive to the state-of-the-art algorithms in terms of both computation time and quality of treatment planning.
\end{abstract}

Key words. Volumetric Modulated Arc Therapy, Greedy algorithm, Gradient projection, Random sampling, Importance sampling.

1. Introduction. Cancer is one of the most deadly diseases, causing millions of deaths all over the world every year. According to the World Cancer Report by the World Health Organization in 2014, about 14.1 million new cases of cancer occurred globally in 2012. It caused about 8.2 million deaths or $14.6 \%$ of all human deaths. The data from United States National Cancer Institute indicates that an estimated 1,658,370 new cases of cancers is diagnosed in the United States and 589,430 people die from these diseases in 2015. Therefore, cancer prevention, diagnosis and treatment are of hyper importance to the world.

Radiation therapy is frequently used in cancer treatment. It is commonly applied to the cancerous cells because of its ability to control cell growth. Most common cancer types can be treated with radiation therapy to a certain extent. It uses relatively high-energy doses of ionizing radiation to damaging the DNA of cancerous tissues leading to cellular death. In the process of the therapy, a radiation beam is generated from a medical linear accelerator fixed in a gantry that can rotate around the patient so that tumors in the patient can receive radiation from various directions. At each direction, the beam is collimated into desired shapes through a device called multi-leaf collimator (MLC) before hitting the patient. An example of MLC is shown in the left side of Figure11 The leaves of MLC can only move in a certain direction at a certain speed. Furthermore, the beam intensity can only vary within a certain range and change at a certain rate. These mechanical limitations impose a few difficult constraints in designing a suitable treatment plan.

Intensity Modulated Radiation Therapy (IMRT) and Volumetric-Modulated Arc Therapy (VMAT) are two major techniques for radiation therapy. IMRT was proposed by Cedric Yu in [14] as an alternative to tomotherapy and much progress [13, 3, 4] has been made over the years. In IMRT, a few angles are selected in advance and multiple shapes of the aperture at each angle are used. In VMAT, on the other hand, the beam intensities and shapes

\footnotetext{
*School of Mathematical Sciences, Peking University, Beijing, CHINA (1200010726@pku.edu.cn).

${ }^{\dagger}$ Corresponding author. Beijing International Center for Mathematical Research, Peking University, Beijing, CHINA (dongbin@math.pku.edu.cn). Research supported in part by the Thousand Talents Plan of China.

${ }^{\ddagger}$ Beijing International Center for Mathematical Research, Peking University, Beijing, CHINA (wenzw@pku.edu.cn). Research supported in part by NSFC grant 11322109 and by the National Basic Research Project under the grant 2015CB856000.
} 

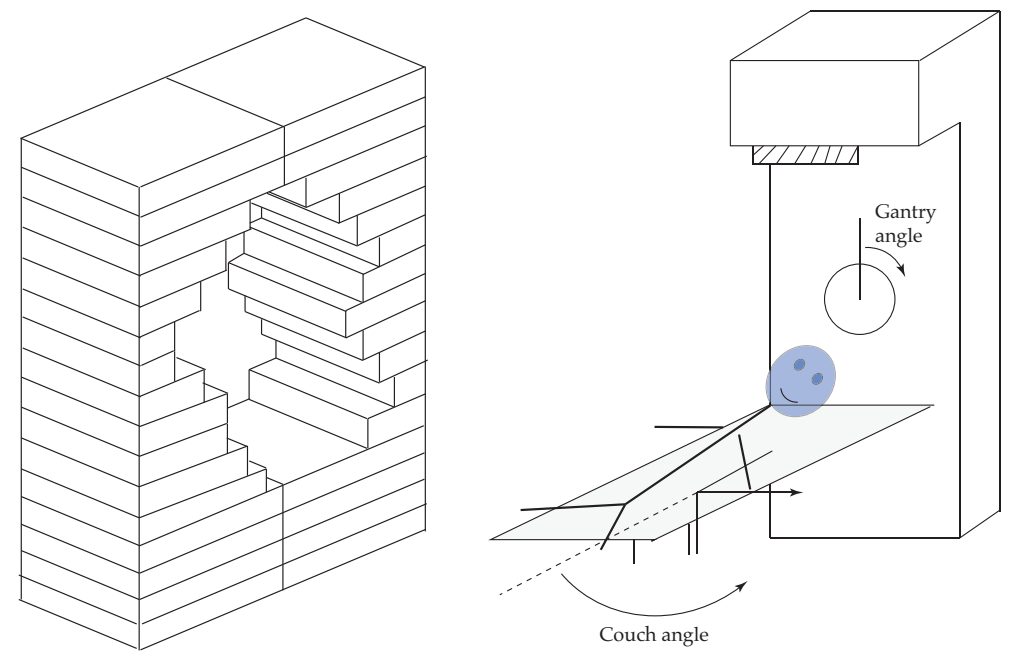

Fig. 1: Left: Diagram of a MLC [6]. Right: The definition of the gantry and the couch angle [7].

of the aperture change continuously and radiation can be delivered to the targets during the whole rotation of the gantry [10]. For some special cases, the couch on which the patient lies can also rotate to fully take advantage of the potentials of VMAT (see Figure 1). Therefore, VMAT is able to significantly reduce the treatment time compared to IMRT without sacrificing treatment quality [8, 12]. Hence, it has experienced an increasing popularity in clinical application [5] in the last few years. However, since VMAT is much more complicated than IMRT because of the flexibility and additional constraints due to mechanical limitations, further improvements of current treatment planning algorithms for VMAT are still needed.

Several models and algorithms have already been developed for the treatment planning in VMAT. The key issue, which is also the main challenge, is to achieve a good balance between delivering sufficient dose to tumors and causing minimal damage to normal tissues and organs. A proper energy functional is often designed based on certain prior information on the patient's anatomy and it offers a proper compromise between the desired dose at the target and the dose at healthy organs. Furthermore, one also needs to consider constraints on the bounds of the beam intensity, an upper bound on the rate of the change of the beam intensity, limit on the moving speed of leaves of the multi-leaf collimator (MLC) and its directionalconvexity. An integer programming problem was formulated in [1] and four methods are proposed: two heuristic strategies based on the Lagrangian relaxation, one heuristic scheme based on a reformulation of the problem, and a metaheuristic strategy based on the guided variable neighborhood scheme. All of these methods can be computationally expensive. A new column-generation-based algorithm was proposed in [11] which was implemented using GPU which significantly improved the efficiency. Nevertheless, their model over-simplified the problem and only a limited number of apertures were considered. In [2], the authors formulated the problem as a nonlinear integer programming problem, and a two-stage algorithm is proposed. More recently, an alternating minimization framework was developed in [6]. The authors use a level-set strategy to represent beam shapes, and a fast sweeping technique is applied to calculate dose intensity. Although their model incorporates most of the main features of VMAT, the computation efficiency of their algorithms still needs to be 
improved.

In this paper, we propose a mixed-integer nonlinear and nonconvex model. The structures in the patient's body are classified into three categories and they are treated differently according to their relative importance. The objective function is constructed as a combination of the quadratic and cubic function, which enables us to achieve a good balance between sufficient dose delivery to the targets and protection of healthy tissues and organs. Due to the physical constraints on the movement of the leaves of MLC, the aperture shapes are characterized by integer variables to describe the directional convexity constraint. Since it is difficult to determine the shapes of the aperture and beam intensities simultaneously, we adopt an iterative alternating minimization framework which solves the model with respect to one variable while the other variable is fixed. The subproblem that determines the shapes of the aperture is combinatorial and is computed using a greedy strategy. Essentially, it revises the boundary of the shapes locally and the elements to be updated are fixed at each iteration. The subproblem that calculates the beam intensities is differentiable and is solved by a standard gradient-projection method using nonmonotone line search with the BB step size. The most computationally intense part of our algorithms is calculating the dose distribution and evaluating the energy function. They are computationally expensive because the number of voxels in the discretization of the energy functional can be up to several millions. Hence, we propose an incremental randomized sampling strategy which only takes a small proportion of the voxels in the energy function and the selection is based on the importance of the voxels. A decremental scheme is also developed to update the boundary of the aperture shapes in the greedy algorithm. Numerical experiments on the prostate and head-and-neck cancer cases in a real medical dataset show that our algorithms can solve the problem more accurately and efficiently than the state-of-the art algorithms. In some cases, the randomized strategy can significantly outperform the deterministic scheme.

The remainder of this paper is organized as follows. In Section 2, we introduce a mathematical model for VMAT and present its discretized form. In Section 3 we develop a greedy algorithm for finding the shapes of the aperture and propose a gradient projection method to optimize beam intensities. The two steps are performed alternately until convergence. Incremental random samplings are introduced to further enhance the performance. Our numerical experiments are demonstrated in Section 4 Finally, we conclude the paper in Section 5.

\section{The VMAT Optimization Model.}

2.1. The Energy Functional. In VMAT, radiation is delivered continuously to the patient in a single rotation. Let the gantry angle $\theta \in[0,2 \pi]$ denote the position of the gantry. For some cases, the couch where the patient lies can also rotate during the procedure. The couch angle can only be chosen in the range of $\left[-\frac{\pi}{2}, \frac{\pi}{2}\right]$. Each time the couch moves to a selected angle, the gantry begins to rotate. Consequently, the entire process can be simplified by assuming that the couch is fixed while the gantry rotates more than one circle. In this case, the angle $\theta$ does not necessarily fall into the range of $[0,2 \pi]$. Let $s(\theta)$ denote the beam intensity at angle $\theta, \Omega(\theta)$ be the aperture formed by the MLC at that angle, and $\hat{y} \in \mathbb{R}^{2}$ be a location in the MLC plane. Then, the dose distribution $z(x)$, with $x \in \mathbb{R}^{3}$, takes the form:

$$
z(x)=\int_{0}^{2 \pi} \int_{\Omega(\theta)} D(x, \hat{y}, \theta) s(\theta) \mathrm{d} \hat{y} \mathrm{~d} \theta,
$$

where $D(x, \hat{y}, \theta)$ is the dose-influence coefficient indicating the dose received at location $x$ in the patient's body when per unit intensity of beamlet radiation is delivered through location $y$ in the MLC plane where the aperture is open. The coefficient $D(x, \hat{y}, \theta)$ is generated from CERR (Computational Environment for Radiotherapy Research) beforehand, and is 
computed specifically for each patient. The aperture shape $\Omega(\theta)$ created by the MLC can be represented equivalently by an indicator function $\psi$ whose value is equal to 1 inside the shape $\Omega(\theta)$ and 0 outside, i.e.,

$$
\psi: \mathbb{R}^{2} \times[0,2 \pi] \rightarrow\{1,0\}, \quad \psi(\hat{y}, \theta)= \begin{cases}1, & (\hat{y}, \theta) \in \Omega(\theta), \\ 0, & (\hat{y}, \theta) \notin \Omega(\theta) .\end{cases}
$$

An illustration of $\psi$ is shown in Figure 2 Therefore, the dose distribution function $z(x)$ can also be expressed as

$$
z(x)=\int_{0}^{2 \pi} \int_{\mathbb{R}^{2}} D(x, \hat{y}, \theta) s(\theta) \psi(\hat{y}, \theta) \mathrm{d} \hat{y} \mathrm{~d} \theta .
$$

The goal of treatment planning for VMAT is to determine the optimal aperture shapes and beam intensities so that the final dose distribution function $z(x)$ is as close to a prescribed treatment plan as possible. Let $S_{r}, r=1, \ldots, n_{S}$, denote different structures in the patient's body which are classified into three categories. The healthy tissues and organs that are very close to the tumors are called critical structures and labeled as the first category $I_{1}$. The cancerous tissues to be eliminated are called target structures and are assigned to $I_{2}$. The remaining tissue types are called remainder structures and will be included in the third category $I_{3}$. Let $m_{r}$ be the maximum dosage that is allowed for the critical and remainder structures, and be the necessary dosage to kill the cancer cells for target tissues, respectively.

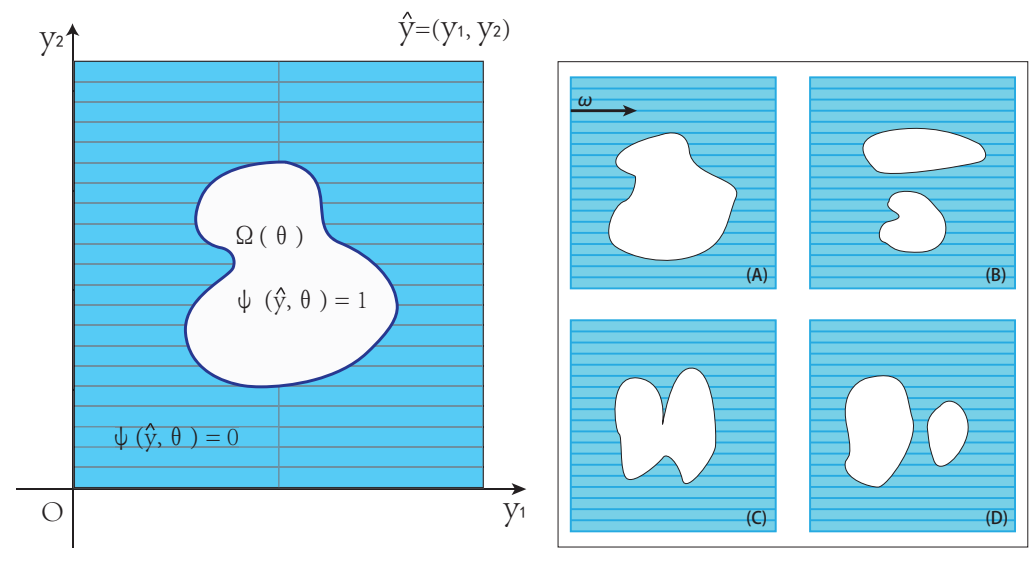

Fig. 2: Left: An aperture at a given gantry angle $\theta$ described by the indicator function $\psi$. Right: An illustration of deliverable apertures ((A) and (B)) and undeliverable ones ((C) and (D)) when leaves are oriented in the $\omega$ direction

We proceed to the definition of an energy functional that measures how far the dose distribution $z(x)$ is from the prescribed treatment plan. For critical and remainder structures, there is no penalty on placing less radiation than the maximum allowable amount. There are only penalties on exceeding the dose limit. On the other hand, for the target structures, there is a penalty as long as the dose administered is not equal to the dose needed. Allowing arbitrary high dose on tumours may seem acceptable at first glance, while it may cause unexpected 
damage on healthy tissues. For a given dose distribution $z(x)$, the aforementioned penalty functional for each structure $S_{r}$ takes the form

$$
F_{r}(z)=\int_{S_{r}} P_{r}(z(x)) \mathrm{d} x
$$

where

(2) $P_{r}(z(x))= \begin{cases}\beta_{r}\left(\max \left\{0, z(x)-m_{r}\right\}\right)^{2}, & r \in I_{1} \cup I_{3}, \\ \alpha_{r}\left(\max \left\{0, m_{r}-z(x)\right\}\right)^{2}+\beta_{r}\left(\max \left\{0, z(x)-m_{r}\right\}\right)^{3}, & r \in I_{2},\end{cases}$

and $\alpha_{r}$ and $\beta_{r}$ are the penalty parameters for the structure $S_{r}$. Using a cubic penalty function on the targets when the dose is over the prescribed limit can often lead to more favorable results in our numerical experiments. The penalty parameters are set as $\alpha_{r}>0$ for $r \in I_{2}$ and $\beta_{r}>0$ for all $r$. The parameters $\alpha_{r}, \beta_{r}$ and $m_{r}$ should be properly adjusted to yield desired plans for different patients.

Combining the definition of $F_{r}(z)$ and $z(x)$, we obtain the following total energy functional with respect to the aperture shapes $\psi(\hat{y}, \theta)$ and beam intensity $s(\theta)$ :

$$
E(\psi, s)=\frac{1}{2} \sum_{r=1}^{n_{S}} \int_{S_{r}} P_{r}\left(\int_{0}^{2 \pi} \int_{\mathbb{R}^{2}} D(x, \hat{y}, \theta) s(\theta) \psi(\hat{y}, \theta) \mathrm{d} \hat{y} \mathrm{~d} \theta\right) \mathrm{d} x .
$$

2.2. The Constraints. We next clarify the constraints imposed by the deliverable aperture shapes and beam intensities due to mechanical limitations. For simplicity, we only consider four major constraints as follows.

(i) The beam intensity $s(\theta)$ should be bounded, i.e., $s \in\left[0, M_{s}\right]$ for a certain given value $M_{s}$.

(ii) Due to the physical restriction on the device, there exists a maximum allowable rate $M$ at which a beam can change its intensity during the rotation. It can be described by the following inequality

$$
\left|\frac{d}{d \theta} s(\theta)\right| \leq M
$$

for all $\theta \in[0,2 \pi]$.

(iii) An aperture is formed when the two sets of MLC leaves move back and forth. Hence, the deliverable aperture shapes must satisfy a directional-convexity requirement. Let $w$ be an unit vector in the direction that the MLC leaves are oriented. The physical constraint requires that $\psi(\hat{y}+\zeta \omega, \theta)=1$ for any $\zeta$ between 0 and $\tau$ if $\psi(\hat{y}, \theta)=1$ and $\psi(\hat{y}+\tau \omega, \theta)=1$ for some $\theta, \tau \in \mathbb{R}$ and $\hat{y}$. An illustration is shown by the image on the right in Figure 2

(iv) The MLC leaves can not move faster than a given speed, or in other words, the aperture shapes cannot change too much between two consecutive angles. Let $M_{A}$ be the maximum speed that the MLC leaves can move. The constraint can be formulated as:

$$
\frac{\left|\left(\nabla \psi \times \omega^{\perp}\right) \cdot \omega\right|}{\left|\nabla \psi \times \omega^{\perp}\right|} \leq M_{A}
$$

where $\omega^{\perp}$ is a unit vector orthogonal to the MLC plane. A simpler discretized formulation of (5) will be used later. 
2.3. The Discretized Model. Now, we describe how the energy functional (3) and the four constraints are discreitized. Recall that $S_{r}, r=1 \ldots, n_{S}$, are domains in $\mathbb{R}^{3}$ that enclose different patient structures. Let $S \subset \mathbb{R}^{3}$ be a cubical computation domain such that $\cup_{r} S_{r} \subseteq$ $S$. We discretize $S$ using a regular grid that divides $S$ into $n_{x}$ voxels which leads to a set of voxels takes the form $\left\{x_{i} \in S: i=1,2, \ldots, n_{x}\right\}$ (see the image on the left in Figure3). Such discretization naturally leads to a discretization of $S_{r}$ as well. With an abuse of terminology, we still denote the discrete voxel set of the $r$-th structure as $S_{r}$ for simplicity. Similarly, we discretize the MLC plane into $n_{y}=n_{y_{1}} n_{y_{2}}$ regular grids, where $n_{y_{1}}$ is the number of grids per row in $y_{1}$ direction, and $n_{y_{2}}$ is the number of grids per line in $y_{2}$ direction. Let $\hat{y}_{j} \in \mathbb{R}^{2}\left(j=1,2, \ldots, n_{y}\right)$ denote the grid point with index $j$ in the MLC plane (see the image on the right in Figure 3). The range-of-rotation of the gantry is discretized into $n_{\theta}$ angles, where $\theta_{k}\left(k=1,2, \ldots, n_{\theta}\right)$ is the $k$-th gantry angle. For convenience, we use $z_{i}, s_{k}$, $\psi_{j k}$ and $D_{i j k}$ to denote $z\left(x_{i}\right), s\left(\theta_{k}\right), \psi\left(\hat{y}_{j}, \theta_{k}\right)$ and $D\left(x_{i}, \hat{y}_{j}, s_{k}\right)$, respectively. The index $i$ is reserved for the voxel index in patient domain $S, j$ is the index of the grid points in aperture domain, and $k$ is the index of gantry angles. Now, the discrete form of $z(x)$ can be written as

$$
z_{i}=\sum_{k=1}^{n_{\theta}} \sum_{j=1}^{n_{y}} D_{i j k} s_{k} \psi_{j k} .
$$

The integration $F_{r}(z)$ on a structure $S_{r}$ can be approximated by a summation of $P_{r}\left(z_{i}\right)$ over all voxels $x_{i}$ in the structure $S_{r}$. Consequently, the total energy functional takes the following discrete form:

$$
E(\psi, s)=\frac{1}{2} \sum_{r=1}^{n_{S}} \sum_{x_{i} \in S_{r}} P_{r}\left(z_{i}\right) .
$$
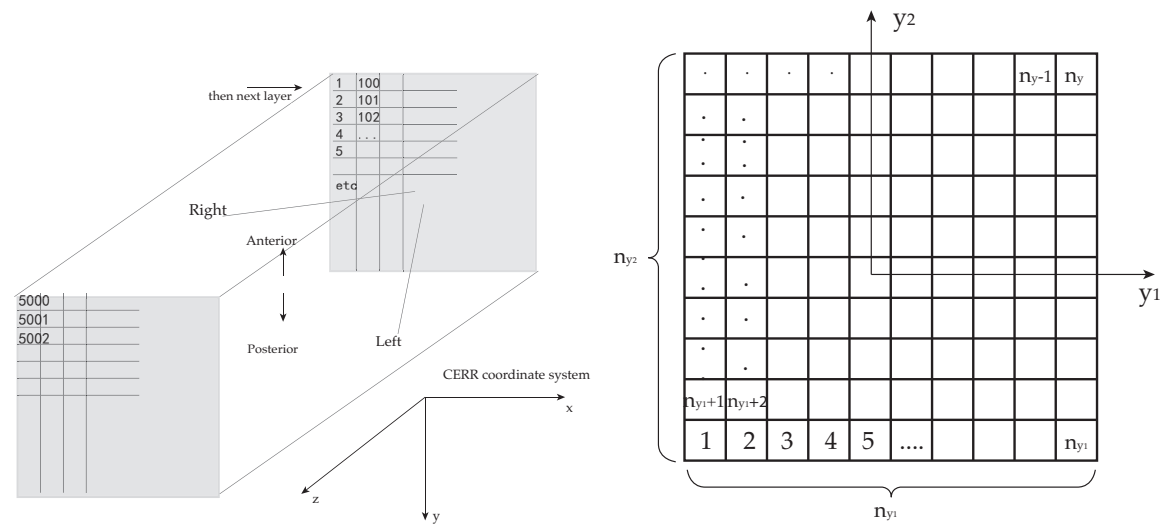

Fig. 3: Left: An illustration of the voxel indices pattern of the patient's body of interest, the patient's orientation and the CERR coordinate system [7]. Right: An illustration of the indices pattern of the discretized MLC plane.

The four constraints mentioned in the previous section can be properly discretized as well. The boundedness of beam intensities $s=\left\{s_{k}: k=1, \ldots, n_{\theta}\right\}$ can be simply written as $0 \leq s_{k} \leq M_{s}$ for all $k$. The constraint (4) can be discretized as

$$
\left|s_{k+1}-s_{k}\right| \leq M\left(\theta_{k+1}-\theta_{k}\right) .
$$


In order to properly discretize the directional convexity constraint and (5), we use an alternative representation of the binary level set function $\psi(\hat{y}, \theta)$. Since the deliverable aperture shapes have to satisfy the direction-convexity and the MLC leaves can only move in $y_{1}$ direction, the aperture can be described by the positions of the first and the last grids that are opened per row, which are essentially the tips of the left and right sets of MLC leaves. Let $L_{k, l}$ and $R_{k, l}$ be the indices of the first (from left to right) and the last open grid (i.e., where the aperture is open) in the $l$-th row of the MLC plane at the $\theta_{k}$ angle. It is not hard to see that any deliverable shape $\psi$ can be characterized equivalently by

$$
(L, R):=\left\{\left(L_{k, l}, R_{k, l}\right) \mid k=1, \ldots, n_{\theta}, l=1, \ldots, n_{y_{2}}\right\} .
$$

Then, one can easily rewrite $z_{i}$ (defined in (6) ) as

$$
z_{i}=\sum_{k=1}^{n_{\theta}} \sum_{l=1}^{n_{y_{2}}} \sum_{j=L_{k, l}}^{R_{k, l}} D_{i j k} s_{k} .
$$

Then, the direction-convexity on the $l$-th row is simply

$$
(l-1) n_{y_{1}} \leq L_{k, l} \leq R_{k, l} \leq \ln _{y_{1}}, \quad L_{k, l}, R_{k, l} \in \mathbb{Z} .
$$

The constraint on the leaf speed limit (5) can be discretized as

$$
\frac{\left|L_{k, l}-L_{k+1, l}\right|}{\theta_{k+1}-\theta_{k}} \leq M_{A}, \frac{\left|R_{k, l}-R_{k+1, l}\right|}{\theta_{k+1}-\theta_{k}} \leq M_{A} .
$$

Therefore, the discretized VMAT optimization problem can be written in the following form:

$$
\begin{array}{ll}
\min _{L, R, s} & E(L, R, s)=\frac{1}{2} \sum_{r=1}^{n_{S}} \sum_{x_{i} \in S_{r}} P_{r}\left(z_{i}\right), \\
\text { s.t. } \quad & z_{i}=\sum_{k=1}^{n_{\theta}} \sum_{l=1}^{n_{y_{2}}} \sum_{j=L_{k, l}}^{R_{k, l}} D_{i j k} s_{k}, \quad i=1, \ldots, n_{x}, \\
& \frac{\left|L_{k, l}-L_{k+1, l}\right|}{\theta_{k+1}-\theta_{k}} \leq M_{A}, \quad k=1, \ldots, n_{\theta}-1, l=1, \ldots, n_{y_{2}}, \\
& \frac{\left|R_{k, l}-R_{k+1, l}\right|}{\theta_{k+1}-\theta_{k}} \leq M_{A}, \quad k=1, \ldots, n_{\theta}-1, l=1, \ldots, n_{y_{2}}, \\
& (l-1) n_{y_{1}} \leq L_{k, l} \leq R_{k, l} \leq l n_{y_{1}}, \quad k=1, \ldots, n_{\theta}, l=1, \ldots, n_{y_{2}}, \\
& L_{k, l}, R_{k, l} \in\left\{1,2, \ldots, n_{y_{1}}\right\}, \quad k=1, \ldots, n_{\theta}, l=1, \ldots, n_{y_{2}}, \\
& \left|s_{k+1}-s_{k}\right| \leq M\left(\theta_{k+1}-\theta_{k}\right), \quad k=1, \ldots, n_{\theta}-1, \\
& s_{k} \in\left[0, M_{s}\right], \quad k=1, \ldots, n_{\theta} .
\end{array}
$$

The model (8) is a mixed-integer nonlinear and nonconvex optimization problem with respect to the aperture shapes $(L, R)$, which are integer variables, and the beam intensities $s=\left\{s_{k}\right.$ : $\left.k=1, \ldots, n_{\theta}\right\}$, which are continuous variables. Note that $z$ is an intermediate variable introduced for convenience.

3. Algorithms For VMAT Optimization. Since solving the VMAT optimization problem (8) simultaneously with respect to both the aperture $(L, R)$ and the beam intensity $s$ can 
be difficult, we adopt a simple alternating minimization framework. At each iteration, the aperture shapes are updated by fixing the beam intensity; then the beam intensity is updated while aperture shapes are fixed. This procedure is repeated until convergence. To further reduce computation cost and improve quality of the results, certain randomized strategies are adopted.

3.1. A Greedy Sampling Algorithm For Finding Aperture Shapes. For fixed beam intensities $s=\left\{s_{k}\right\}$, the optimization problem with respect to the aperture shapes is:

$$
\begin{array}{ll}
\min _{L, R} \quad & E(L, R, s)=\frac{1}{2} \sum_{r=1}^{n_{S}} \sum_{x_{i} \in S_{r}} P_{r}\left(z_{i}\right), \\
\text { s.t. } \quad z_{i}=\sum_{k=1}^{n_{\theta}} \sum_{l=1}^{n_{y_{2}}} \sum_{j=L_{k, l}}^{R_{k, l}} D_{i j k} s_{k}, \quad i=1, \ldots, n_{x}, \\
& \frac{\left|L_{k, l}-L_{k+1, l}\right|}{\theta_{k+1}-\theta_{k}} \leq M_{A}, \quad k=1, \ldots, n_{\theta}-1, l=1, \ldots, n_{y_{2}}, \\
& \frac{\left|R_{k, l}-R_{k+1, l}\right|}{\theta_{k+1}-\theta_{k}} \leq M_{A}, \quad k=1, \ldots, n_{\theta}-1, l=1, \ldots, n_{y_{2}}, \\
& (l-1) n_{y_{1}} \leq L_{k, l} \leq R_{k, l} \leq l n_{y_{1}}, \quad k=1, \ldots, n_{\theta}, l=1, \ldots, n_{y_{2}}, \\
& L_{k, l}, R_{k, l} \in\left\{1,2, \ldots, n_{y_{1}}\right\}, \quad k=1, \ldots, n_{\theta}, l=1, \ldots, n_{y_{2}} .
\end{array}
$$

Since solving the above nonconvex mixed-integer programming directly is challenging, we develop a greedy algorithm inspired by the heuristic strategy proposed by [6].

Due to the constraints on the speed limit of the leaves and the directional-convexity of the aperture shapes, the leaf pairs $(L, R)=\left\{\left(L_{k, l}, R_{k, l}\right)\right\}$ cannot change too fast. The basic idea of our algorithm is to only update the values of leaf pair $\left(L_{\hat{k}, \hat{l}}, R_{\hat{k}, \hat{l}}\right)$ one at a time. Given an angle $\theta_{\hat{k}}$ and the amount of movement $\delta=\left(\delta_{1}, \delta_{2}\right)$ of the left and right tips of the $\hat{l}$-th row of the MLC leaves, the next position $(\widehat{L}, \widehat{R})$ can be described by

$$
\widehat{L}_{i j}=\left\{\begin{array}{ll}
L_{\hat{k}, \hat{l}}+\delta_{1}, & \text { if } i=\hat{k} \text { and } j=\hat{l}, \\
L_{i j}, & \text { otherwise, }
\end{array} \widehat{R}_{i j}= \begin{cases}R_{\hat{k}, \hat{l}}+\delta_{2}, & \text { if } i=\hat{k} \text { and } j=\hat{l} \\
R_{i j}, & \text { otherwise. }\end{cases}\right.
$$

There are two types of elementary movements of $\left(\delta_{1}, \delta_{2}\right)$, i.e.,

$$
\Delta_{\hat{k}, \hat{l}}^{L, c}=\left\{(a, b) \mid b=0, a \in \mathbb{Z}_{c}\right\}, \quad \Delta_{\hat{k}, \hat{l}}^{R, c}=\left\{(a, b) \mid a=0, \quad b \in \mathbb{Z}_{c}\right\} .
$$

where $\mathbb{Z}_{c}=\{z \mid-c \leq z \leq c, z \in \mathbb{Z}\}$. A value $\delta \in \Delta_{\hat{k}, \hat{l}}^{L, c}$ means that the left tip of the leaf can move in a small neighborhood of $L_{\hat{k}, \hat{l}}$ with respect to the first index with the right tip fixed, and similarly for $\delta \in \Delta_{\hat{k}, \hat{l}}^{R, c}$. It is easy to verify that these two types of movements can be combined to generate general movements. Hence, we focus on the movements in $\Delta_{\hat{k}, \hat{l}}^{L, c}$ and $\Delta_{\hat{k}, \hat{l}}^{R, c}$. Our aperture algorithm is to move the leaf pair row by row and the changes are accumulated until the optimal shapes are obtained. In fact, the greedy algorithm proposed in [6] is a special case of (11) with $c=1$, while our method has more flexibility in changing the aperture shapes by searching in a larger region defined by (11).

After each movement (10), the change of the energy function can be relatively easily calculated because the update only modifies the dosages on a small portion of the total voxels 
when $c$ is small. For example, if $\delta_{1}>0$ and $\delta_{2}=0$, we have

$$
\hat{z}_{i}=z_{i}-\sum_{j=L_{\hat{k}, \hat{l}}}^{L_{\hat{k}, \hat{\imath}}+\delta_{1}-1} D_{i j \hat{k}} s_{\hat{k}}
$$

which yields

$$
\Delta E_{\hat{k}, \hat{l}}^{\delta}=E(\widehat{L}, \widehat{R}, s)-E(L, R, s)=\frac{1}{2} \sum_{r=1}^{n_{S}} \sum_{x_{i} \in S_{r}, \hat{z}_{i} \neq z_{i}}\left(P_{r}\left(\hat{z}_{i}\right)-P_{r}\left(z_{i}\right)\right) .
$$

Similar relationships hold for other values of $\delta$. When $c$ is set to 1 in (11), the number of operations needed to determine $\Delta E_{\hat{k}, \hat{l}}$ is of the order of the number of $i$ such that $D_{i j \hat{k}} \neq 0$. Consequently, the best possible local modification of the left tip is a movement $\delta \in \Delta_{\hat{k}, \hat{l}}^{L, c}$ such that $\Delta E_{\hat{k}, \hat{l}}^{\delta} \leq 0$ and the resulting position $(\hat{L}, \hat{R})$ represents a deliverable aperture shape. The best one from the right side of the leaf can be found in the same fashion. For convenience, we denote these two movements as follows:

$$
\begin{gathered}
\delta_{\hat{k}, \hat{l}}^{L}=\arg \min _{\delta}\left\{\Delta E_{\hat{k}, \hat{l}}^{\delta} \mid \delta \in \Delta_{\hat{k}, \hat{l}}^{L, c}, \quad \Delta E_{\hat{k}, \hat{l}}^{\delta} \leq 0, \quad(\hat{L}, \hat{R}) \text { is feasible }\right\} \\
\delta_{\hat{k}, \hat{l}}^{R}=\arg \min _{\delta}\left\{\Delta E_{\hat{k}, \hat{l}}^{\delta} \mid \delta \in \Delta_{\hat{k}, \hat{l}}^{R, c}, \quad \Delta E_{\hat{k}, \hat{l}}^{\delta} \leq 0, \quad(\hat{L}, \hat{R}) \text { is feasible }\right\} .
\end{gathered}
$$

Note that the values of $\delta_{\hat{k}, \hat{l}}^{L}$ and $\delta_{\hat{k}, \hat{l}}^{R}$ might be zero if there is no better position than $(L, R)$.

We next present the rules on selecting elements $(\hat{k}, \hat{l})$ to update sequentially. In order to save on computation time, we create a heap at the beginning and only update elements in the heap based on the following strategies. Denote the collection of all feasible movements that lead to a strict decreasing of the energy function from the current aperture shape by

$$
\Gamma:=\left\{(\hat{k}, \hat{l}, \delta) \mid \Delta E_{\hat{k}, \hat{l}}^{\delta}<0, \hat{k}=1, \ldots, n_{\theta}, \hat{l}=1, \ldots, n_{y_{2}}, \delta \in\left\{\delta_{\hat{k}, \hat{l}}^{L}, \delta_{\hat{k}, \hat{l}}^{R}\right\}\right\} .
$$

Then we sort all elements in $\Gamma$ so that the corresponding values $\Delta E_{\hat{k}, \hat{l}}^{\delta}$ are in an ascending order and we still denote the sorted list as $\Gamma$. Obviously, the first element in $\Gamma$ has the smallest $\Delta E_{\hat{k}, \hat{l}}^{\delta}$ and it is the best local refinement in $\Gamma$. We update the aperture shape using this element and delete it from the heap $\Gamma$ afterwards. Since this change may violate the feasibility of other values in $\Gamma$, we recompute $\Delta E_{\hat{k}, \hat{l}}^{\delta}$ for the next $\min \left(\Upsilon,\left|\Gamma_{\Pi}\right|\right)$ elements in line in $\Gamma$ and fix their ordering. The value of $\Upsilon$ is usually set to 5 to 10 for saving the computational cost. This procedure is repeated and iterated until $\Gamma$ is empty.

The major computational cost of the above greedy procedure is calculating $\Delta E_{\hat{k}, \hat{l}}^{\delta}$ repeatedly for all $\hat{k}=1, \ldots, n_{\theta}$ and $\hat{l}=1, \ldots, n_{y_{2}}$. However, since only one row of the aperture shapes for a specific value of $\delta$ is updated at each iteration, computing all possible $\Delta E_{\hat{k}, \hat{l}}^{\delta}$ in the heap $\Gamma$ is definitely not the best strategy. If we randomly take a small number of leaf pairs, the computational cost can be significantly reduced. The randomness in sampling may also prevent the algorithm from being trapped in a local minimum at an early stage. To be more precise, given a sampling ratio $0<\kappa_{\Pi} \leq 1$, we take $\kappa_{\Pi}$ uniformly sampled elements from $\Gamma$ as

$$
\Gamma_{\Pi}:=\{(\hat{k}, \hat{l}, \delta) \mid(\hat{k}, \hat{l}, \delta) \in \Gamma\} \text { and }\left|\Gamma_{\Pi}\right|=\kappa_{\Pi}|\Gamma|
$$


Then the randomized greedy algorithm only updates elements in the set $\Gamma_{\Pi}$. In our experiments, the amount of change of aperture shapes decreases quickly for each iteration. Therefore, we start with fully sampling $\kappa_{\Pi}=1$ and gradually decrease the sampling rate at each iteration.

We outline our aperture optimization algorithm as follows.

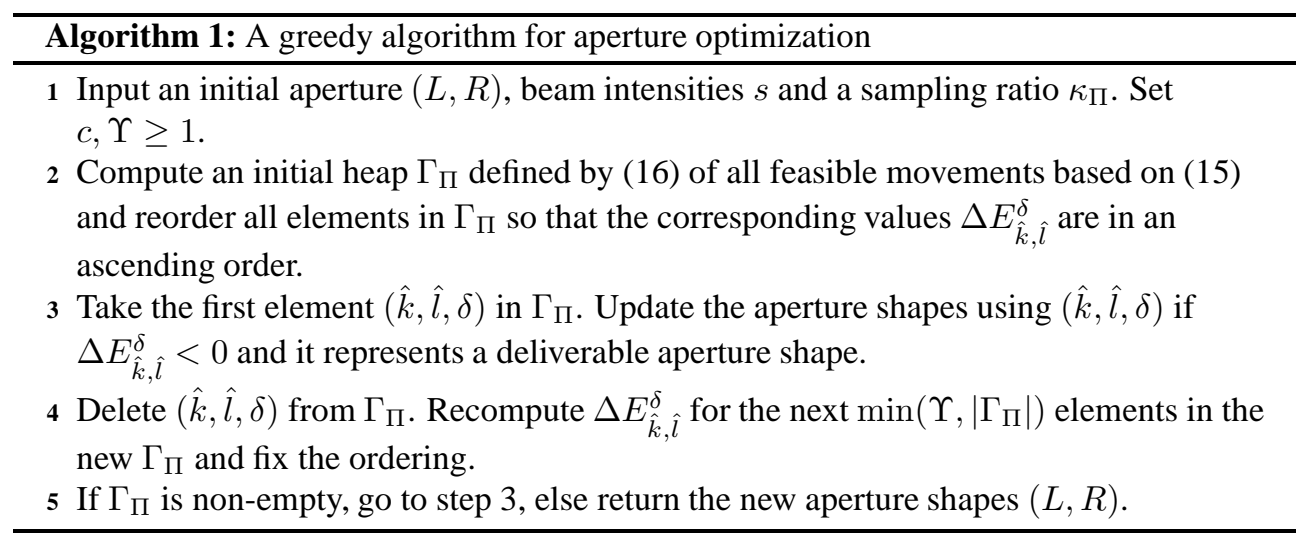

3.2. Projected Gradient Methods for Beam Intensity Optimization. For fixed aperture shapes at different angles $(L, R)$, the optimization problem with respect to the beam intensities $s$ is:

$$
\begin{array}{ll}
\min _{s} & E(L, R, s)=\frac{1}{2} \sum_{r=1}^{n_{S}} \sum_{x_{i} \in S_{r}} P_{r}\left(z_{i}\right), \\
\text { s.t. } & z_{i}=\sum_{k=1}^{n_{\theta}} \sum_{l=1}^{n_{y_{2}}} \sum_{j=L_{k, l}}^{R_{k, l}} D_{i j k} s_{k}, \quad i=1, \ldots, n_{x}, \\
& \left|s_{k+1}-s_{k}\right| \leq M\left(\theta_{k+1}-\theta_{k}\right), \quad k=1, \ldots, n_{\theta}-1, \\
& s_{k} \in\left[0, M_{s}\right], \quad k=1, \ldots, n_{\theta} .
\end{array}
$$

The main difficulty in solving (17) is the nonlinearity of the objective function $E(L, R, s)$. Since the constraints are relatively simple, we apply the widely used projected gradient method to solve (17).

The partial derivative of the energy function with respect to $s_{k}$ is given as follows:

$$
\frac{\partial E(L, R, s)}{\partial s_{k}}=\frac{1}{2} \sum_{r=1}^{n_{S}} \sum_{x_{i} \in S_{r}} P_{r}^{\prime}\left(z_{i}\right) \frac{\partial z_{i}}{\partial s_{k}}=\frac{1}{2} \sum_{r=1}^{n_{S}} \sum_{x_{i} \in S_{r}} P_{r}^{\prime}\left(z_{i}\right) d_{i k},
$$

where $d_{i k}=\sum_{l=1}^{n_{y_{2}}} \sum_{j=L_{k, l}}^{R_{k, l}} D_{i j k}$, and

$$
P_{r}^{\prime}(z)= \begin{cases}2 \beta_{r}\left(\max \left\{0, z-m_{r}\right\}\right), & r \in I_{1} \cup I_{3}, \\ 2 \alpha_{r}\left(\max \left\{0, m_{r}-z\right\}\right)+3 \beta_{r}\left(\max \left\{0, z-m_{r}\right\}\right)^{2}, & r \in I_{2}\end{cases}
$$

Denote $s^{(q)}$ as the approximated optimal intensity values at the $q$-th iteration, and $\nabla_{s} E^{(q)}:=$ $\nabla_{s} E\left(L, R, s^{(q)}\right)$. Given $s^{(q)}$ and a step size $\tau^{(q)}$, the projected gradient method first approximates the energy function by linearizing it with respect to $s$ and adding a proximal term 
as

$$
\begin{aligned}
& E\left(L, R, s^{(q)}\right)+\left(\nabla_{s} E^{(q)}\right)^{\top}\left(s-s^{(q)}\right)+\frac{1}{2 \tau^{(q)}}\left\|s-s^{(q)}\right\|^{2} \\
= & \frac{1}{2 \tau^{(q)}}\left\|s-\left(s^{(q)}-\tau^{(q)} \nabla_{s} E^{(q)}\right)\right\|^{2}+\text { constant }
\end{aligned}
$$

then computes a new trial point $s\left(\tau^{(q)}\right)$ as the optimal solution of

$$
\begin{array}{ll}
\min _{s} & \frac{1}{2}\left\|s-\left(s^{(q)}-\tau^{(q)} \nabla_{s} E^{(q)}\right)\right\|_{2}^{2}, \\
\text { s.t. } & \left|s_{k+1}-s_{k}\right| \leq M\left(\theta_{k+1}-\theta_{k}\right), \quad k=1, \ldots, n_{\theta}-1, \\
& s_{k} \in\left[0, M_{s}\right], \quad k=1, \ldots, n_{\theta} .
\end{array}
$$

The subproblem (18) is a standard quadratic problem whose objective function and constraints are simple. Since the length $n_{\theta}$ is often relatively small, (18) can be solved efficiently by commercial solvers such as Mosek.

Another key algorithmic issue is the determination of a suitable step size $\tau^{(q)}$. Instead of using the classical Armijo-Wolfe based monotone line search, we apply the nonmonotone line search determined by the BB formula, which is proposed by Barzilai and Borwein [9] in 1988. We have found that the BB method is more efficient than the monotone line search for our problem. At iteration $q$, the step size is computed by

$$
\tau_{B B_{1}}^{(q)}=\frac{\left(\nu^{(q-1)}\right)^{T} y^{(q-1)}}{\left(y^{(q-1)}\right)^{T} y^{(q-1)}}, \quad \tau_{B B_{2}}^{(q)}=\frac{\left(\nu^{(q-1)}\right)^{T} \nu^{(q-1)}}{\left(\nu^{(q-1)}\right)^{T} y^{(q-1)}}
$$

where $y^{(q-1)}=\nabla_{s} E^{(q)}-\nabla_{s} E^{(q-1)}$ and $\nu^{(q-1)}=s^{(q)}-s^{(q-1)}$. In order to guarantee convergence, the final value of $\tau^{(q)}$ is a fraction of $\tau_{B B_{1}}^{(q)}$ and $\tau_{B B_{2}}^{(q)}$ determined by a nonmonotone search condition in [15]. Let $C^{(0)}=E\left(L, R, s^{(0)}\right), V^{(q+1)}=\eta V^{(q)}+1$ and $V^{(0)}=1$. The new points are generated iteratively in the form $s^{(q+1)}=s\left(\tau^{(q)}\right)$, where $s\left(\tau^{(q)}\right)$ is the solution of subproblem (18), $\tau^{(q)}=\sigma^{t} \tau_{B B_{1}}^{(q)}$ or $\tau^{(q)}=\sigma^{t} \tau_{B B_{2}}^{(q)}, \sigma \in(0,1)$ and $t$ is the smallest nonnegative integer satisfying

$$
E\left(L, R, s^{(q+1)}\right) \leq C^{(q)}-\frac{\gamma}{2}\left\|s^{(q+1)}-s^{(q)}\right\|_{F}^{2},
$$

where $\gamma>0$, each reference value $C^{(q+1)}$ is taken to be the convex combination of $C^{(q)}$ and $E\left(L, R, s^{(q+1)}\right)$ as $C^{(q+1)}=\left(\eta V^{(q)} C^{(q)}+E\left(L, R, s^{(q+1)}\right)\right) / V^{(q+1)}$. Since we observe that the BB step size $\tau_{B B 1}^{(q)}$ or $\tau_{B B 2}^{(q)}$ is often sufficient for (20) to hold in our numerical experiments, we set the initial value of $\tau^{(q)}$ as

$$
\tau^{(q)}= \begin{cases}\tau_{B B 1}^{(q)}, & q \text { is odd } \\ \tau_{B B 2}^{(q)}, & q \text { is even }\end{cases}
$$

Our method on finding the beam intensities is presented in Algorithm 2, We terminate the algorithm when the change of the energy function is small comparing to the initial value $E\left(L, R, s^{(0)}\right)$, i.e.,

$$
\left|E\left(L, R, s^{(q+1)}\right)-E\left(L, R, s^{(q)}\right)\right| \leq \epsilon_{b}\left|E\left(L, R, s^{(0)}\right)\right|,
$$


where $\epsilon_{b}$ is a small positive number.

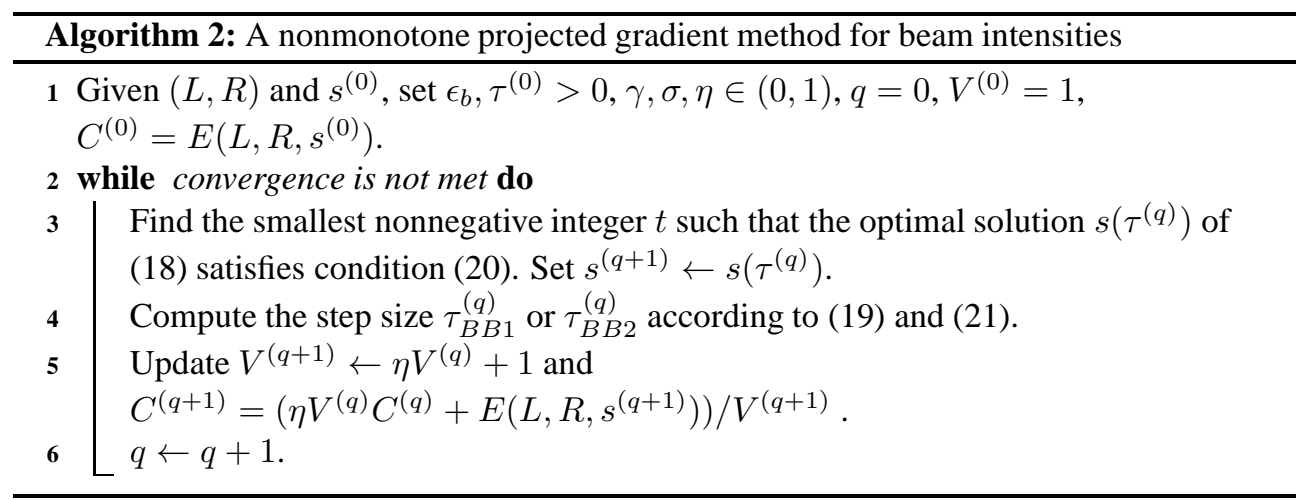

3.3. Incremental Importance Sampling in Computing the Energy Function. Since the number $n_{x}$ of voxels can easily be as large as several millions, calculating the dose distribution $z$ and evaluating the energy function $E(L, R, s)$ are computationally expensive in Algorithms 1 and 2. However, the similarity among the same type of tissues and organs often leads to a large amount of uniformity in the measurements. This indicates that a full evaluation of $E(L, R, s)$ and its gradient $\nabla_{s} E(L, R, s)$ may not be necessary to make sufficient progress in solving (8). This observation motivates our incremental importance sampling strategy, which only evaluates the objective function $E(L, R, s)$ with respect to a small number of carefully selected voxels. Comparing to the classical incremental randomized algorithms, our selection of the voxels is based on the importance of the voxels measured by certain rules. The cost of incremental importance sampling methods is proportional to the full size methods. In order to eventually achieve a high accuracy, the sampling ratio is increased gradually as the number of iteration increases.

We now introduce the details on how the voxels are chosen. Generally speaking, remainder structures are not as important as critical or target structures in the treatment planning. It is reasonable to treat these voxels in the remainder structures as equally important. Also, eliminating the target structures and protecting healthy tissues and organs are equally important. For voxels in targets and tissues/organs, we define two different importance metrics respectively. For target structures, the penalty function $P_{r}\left(z_{i}\right)$ defined in (2) is a reasonably good metric since it measures how much each voxel contributes to the total energy. This function is nonzero on almost every voxels in these structures. For critical structures, using $P_{r}\left(z_{i}\right)$ may not be the best option because voxels in healthy tissues/organs that receive dosage below $m_{r}$ have a penalty value $P_{r}\left(z_{i}\right)=0$ and these voxels may be the majority of voxels of critical structures. The voxels with the same penalty value will be considered equally important and sampled with the same probability. Certainly, those who receive more dosage are in a more dangerous position and we should pay more attention on them. Hence, the ratio between the dosage $z_{i}$ and the maximum dosage can be a good indicator of importance since it provides a larger weight to the voxels with a higher dosage and $P_{r}\left(z_{i}\right)=0$. Note that a portion of the voxels may still receive zero dosage, we further add a small number $h>0$ and assign a metric $\frac{z_{i}}{m_{r}}+h$ to each voxel.

Let $\widehat{S}_{u}=\cup_{r \in I_{u}} S_{r}, u=1,2,3$. For a specifically given dose distribution $z^{*}$, the proba- 
bility of choosing a voxel $x_{i} \in S_{r}$ is defined as

$$
\pi_{r, i}^{z^{*}}= \begin{cases}\frac{\frac{z_{i}^{*}}{m_{r}}+h}{\sum_{x_{i} \in \widehat{S}_{1}}\left(\frac{z_{i}^{*}}{m_{r}}+h\right)}, & r \in I_{1}, \\ \frac{P_{r}\left(z_{i}^{*}\right)}{\sum_{x_{i} \in \widehat{S}_{2}} P_{r}\left(z_{i}^{*}\right)}, & r \in I_{2}, \\ \frac{1}{\left|\widehat{S}_{3}\right|}, & r \in I_{3},\end{cases}
$$

where $\left|\widehat{S}_{u}\right|$ is the total number of voxels in $\widehat{S}_{u}$. Let $0<\kappa_{u} \leq 1$ be the sampling ratio such that $\kappa_{u}$ is gradually increased in critical and target structures while $\kappa_{u}$ is fixed in remainder structures. The voxels in $\widehat{S}_{u}$ are chosen in $c=\kappa_{u}\left|\widehat{S}_{u}\right|$ independent identical trials where in each trial the $i$-th voxel in $S_{r}$ is selected with probability $\pi_{r, i}^{z^{*}}$, and the collection of the corresponding indices is denoted by $\mathcal{B}_{r}^{z^{*}}$. In particular, the samples are chosen without replacement (i.e., every voxel occurs only once).

Therefore, the sampled total energy functional is defined as

$$
E_{z^{*}}(L, R, s)=\sum_{u=1}^{3} \frac{1}{\kappa_{u}}\left(\sum_{r \in I_{u}} \sum_{i \in \mathcal{B}_{r}^{z^{*}}} P_{r}\left(z_{i}\right)\right) .
$$

Finally, we obtain the randomized version of Algorithms 1 and 2 by simply replacing $E(L, R, s)$ by the its sampled version $E_{z^{*}}(L, R, s)$. Consequently, the computational time is greatly reduced when the sampling ratio $\kappa_{u}$ is small.

3.4. Ramdomized Algorithms for VMAT Optimization. Combining the algorithms for finding the aperture shapes and beam intensities together, we present our randomized VMAT optimization method in Algorithm 3 . With an abuse of terminology, the superscripts of beam intensities for the inner iteration $s^{(q)}$ in Algorithm 2 and the outer iteration $s^{(n)}$ in Algorithm 3 are both used whose meaning should be clear from context. We terminate the algorithm when the relative change on the value of the energy function is small, i.e.,

$$
\left|E_{z^{(n+1)}}\left(L^{(n+1)}, R^{(n+1)}, s^{(n+1)}\right)-E_{z^{(n)}}\left(L^{(n)}, R^{(n)}, s^{(n)}\right)\right| \leq \epsilon\left|E_{z^{(n)}}\left(L^{(n)}, R^{(n)}, s^{(n)}\right)\right|,
$$


where $\epsilon$ is small positive number. Note that Algorithm 3 with $\kappa_{\Pi}=1$ and $\kappa_{u}=1$ reduces to a deterministic method without any sampling.

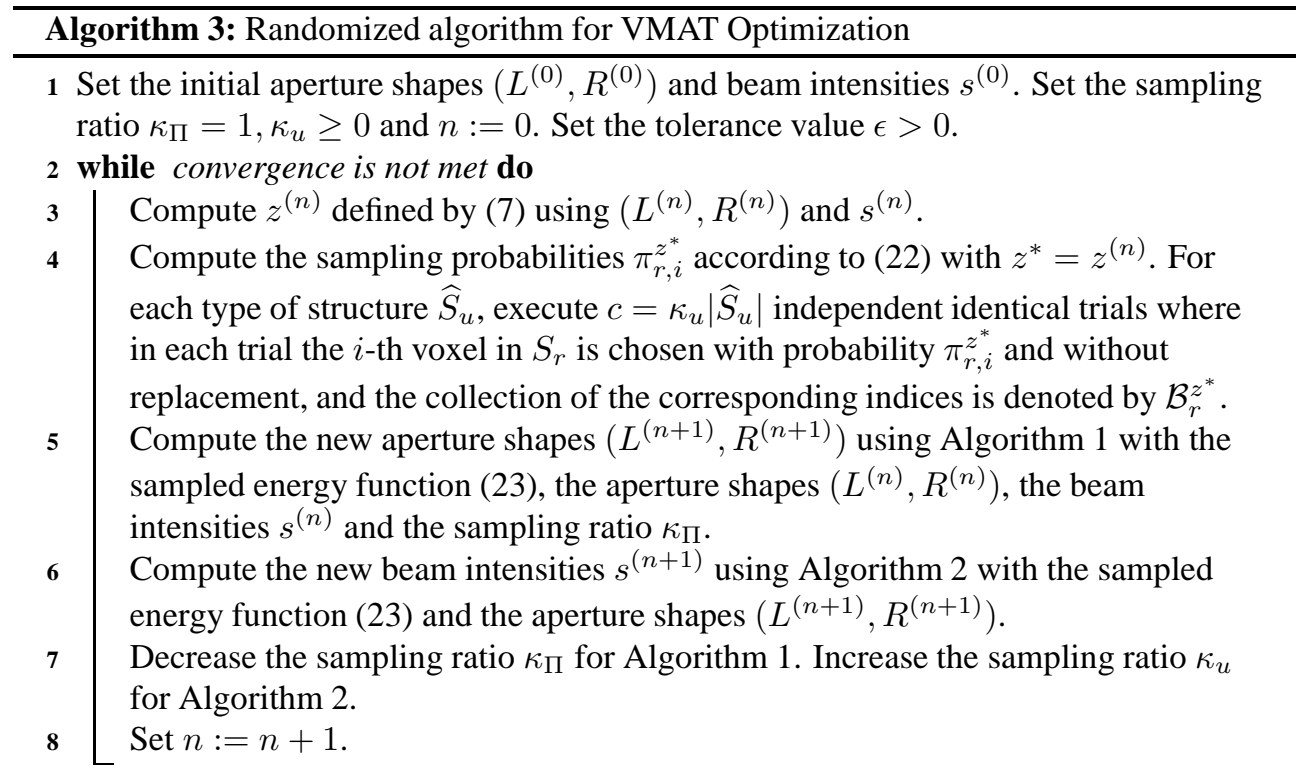

The Algorithm 3 can be further improved in a few ways. For example, we have found that the beam intensity algorithm can be time consuming. The dose distribution changes drastically when the beam intensity changes, which means that one have to recalculate the full dose distribution in every iteration of Algorithm 2, On the other hand, only a small part of the dose distribution changes when the leaves changes in Algorithm 1 Hence, if the change of the aperture shapes is not large, we can skip the step of updating beam intensities and continue refining the aperture shapes.

Although the solutions yielded by Algorithm 3 will not be exactly the same for every run due to the random sampling, they are still similar to each other. The algorithm is robust since the increase of the sampling ratios on critical and target structures enables the algorithm to eventually take all the voxels into account. In our experiments, the randomized algorithm often returns a solution with better quality and uses less amount of time than the deterministic algorithms.

4. Numerical results. In this section, we evaluate the performance of our algorithms on two examples, prostate and head-and-neck cancer, from the common optimization for radiation therapy (CORT) datase *. More detailed information on the CORT dataset can be found in [7]. We have implemented the method in [6] and our Algorithm[3] using C. The algorithm introduced by [6] shall be referred to as "GEAltMin" (Greedy-Euler-flow-AlternatingMinimization). The deterministic version of Algorithm 3 with $\kappa_{\Pi}=1$ and $\kappa_{u}=1$ will be called "GGAltMin" (Greedy-Gradient-projetion-Alternating-Minimization). The randomized version of Algorithm 3 is called "RGAltMin" (Randomized-Greedy-Gradient-projetionAlternating-Minimization). Our numerical experiments on the comparisons of these three algorithms are preformed on a workstation with two twelve-core Intel Xeon E5-2697 CPUs and $128 \mathrm{~GB}$ of memory running Ubuntu 12.04 . The penalty parameters in the VMAT models are tuned for the best results.

The quality of the computed treatment plans are evaluated through the so-called Dose-

*Downloadable from http://gigadb.org/dataset/100110 
Volume histogram (DVH). For a given structure $S_{r}$, a dose distribution $z(x)$ and the corresponding bound $m_{r}$, the DVH function for this structure is defined as the percentage of the structure that is radiated at or above the dose level $m_{r}$, i.e.,

$$
\operatorname{DVH}\left(m_{r}\right)=\frac{\operatorname{vol}\left(\left\{x \mid z(x) \geq m_{r}\right\}\right)}{\operatorname{vol}\left(S_{r}\right)},
$$

where $\operatorname{vol}\left(S_{r}\right)$ denotes the volume of the structure $S_{r}$. For a target structure, the optimal DVH curve should remain $100 \%$ before the prescribed dose and drop sharply to $0 \%$ afterwards. For a critical structure, the desired DVH curve should stay $0 \%$ above the safe dose limit for the particular tissue involved.

We first test the prostate case. A structure named PTV_68 is set as the target, which is a geometric expansion of the gross tumor volume (GTV). For this region, a 73.8 Gy radiation dose is prescribed to eliminate the cancerous growth. For the nearby critical structures of the bladder and rectum, safe dose limits are $23.52 \mathrm{~Gy}$ and $32.92 \mathrm{~Gy}$ respectively. A summary of computational results is presented in Table 1 , where "iteration" is the total number of the outer iterations for each algorithm, " $E$ " denotes the value of the energy function and "runtime" is the runtime measured in seconds. Since there are randomness in the run of RGAltMin, its reported results are the average of 20 runs. The table shows that our algorithms take much fewer iterations and much less time to achieve a comparable objective function value. Although the iteration number of RGAltMin is more than that of GGAltMin, the former is still faster than the later because the cost per iteration is cheaper due to the sampling strategies.

Figure 5 shows the comparisons between the three algorithms. The dose distribution and their level-sets overlaying the outlined prostate and other organs on a particular slice of the patient's scan is given in the first row of this figure. The red color means that the dose amount is high and the blue color suggests that the dose amount is low. The second row of this figure depicts the DVH curves. A summary of DVH is further shown in Table 2 In general, the DVH should be low on the critical strucutures Bladder and rectrum. The smaller these values are, the better the algorithm is. On the other hand, a higher DVH is desired on the target PTV_68. We can observe that the dosage is high on target structures and it is low on critical structures. In particular, the curve of RGAltMin shows that $95.35 \%$ of the target is dosed at or above the required level, and $74.19 \%$ of the bladder and $76.70 \%$ of the rectum are dosed below their safe limit in critical structures. The curve of GGAltMin shows that $94.87 \%$ of the target is dosed at or above the required level and $73.17 \%$ of the bladder and $75.36 \%$ of the rectum are dosed below their safe limit in critical structures. For GEAltMin, only $70 \%$ of the bladder and $75 \%$ of the rectum are dosed below the safe limit respectively. The computational results of the 20 runs of GEAltMin are further shown in Figure 4 using box plots. GEAltMin are better than the other two algorithms in most cases and it is highly competitive to GGAltMin even in the worst case. These figures and tables verify that the randomized algorithm RGAltMin performs best in terms of the DVH quality and it is robust. Finally, some samples of the aperture shapes and beam intensities for the VMAT plans computed by GGAltMin is shown in the left side of Figure 8 .

\begin{tabular}{cccr}
\hline algorithm & iteration & $\mathrm{E}$ & runtime \\
\hline GEAltMin & 200 & $8.50 e 8$ & $51 \mathrm{~m} 1 \mathrm{~s}$ \\
GGAltMin & 43 & $8.24 e 8$ & $9 \mathrm{~m} 33 \mathrm{~s}$ \\
RGAltMin & 64 & $8.20 e 8$ & $8 \mathrm{~m} 17 \mathrm{~s}$ \\
\hline
\end{tabular}

Table 1: A summary of computational results on the prostate case. 


\begin{tabular}{cccc}
\hline algorithm & Bladder & Rectum & PTV_68 \\
\hline GEAltMin & $28.20 \%$ & $30.34 \%$ & $95.33 \%$ \\
GGAltMin & $26.83 \%$ & $24.64 \%$ & $94.87 \%$ \\
RGAltMin & $25.91 \%$ & $23.30 \%$ & $95.35 \%$ \\
\hline
\end{tabular}

Table 2: A summary of DVH on the prostate case.
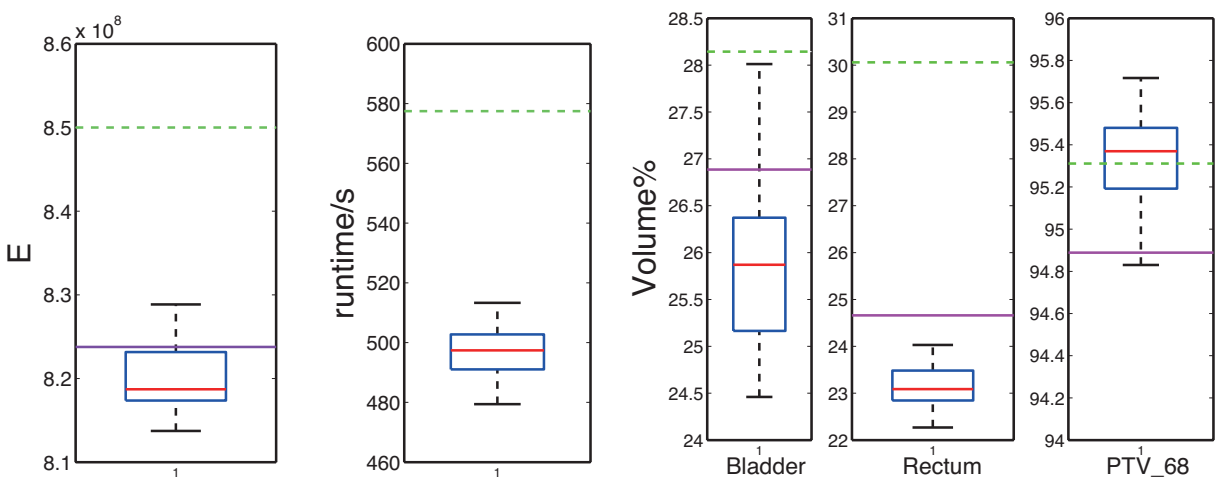

Fig. 4: Boxplots of the 20 runs of RGAltMin on the prostate case. The figures from left to right are corresponding to energy, runtime and DVHs of Bladder, Rectum and PTV_68, respectively. The green dashed lines and the purple solid lines represent the corresponding values yielded by GEAltMin and GGAltMin, respectively. The runtime of the GEAltMin is not shown in the second figure since it is way larger than 600 seconds.

The second test is on the head-and-neck case, which is much more complicated than the prostate case. It involves two different targets, labeled as PTV_70 and PTV_63, respectively. PTV_70 is expanded on the GTV. It consists of two components of the tumors, and a dose of 70 Gy is prescribed. PTV_63 is a larger target area which contains nearby high-risk region. A dose of $63 \mathrm{~Gy}$ is prescribed for this target. Two critical structures are the parotid and spinal cord PRV, which have dose limits of 40Gy and 45Gy, respectively. The head-and-neck case is complicated because of the number of targets, the relative positions as well as the volumes of the target and critical structures. The parotid is close to PTV_63 and much smaller compared to PTV_63. If the DVH of PTV_63 is increased, the dose on parotid will also be increased inevitably. Due to the small size of the parotid, violating its dose limit may only slightly increase the value of the energy function. Hence, an algorithm may sacrifice the parotid and fail to yield the desired result. If the penalty parameter $\beta_{r}$ for the parotid is set large enough, it will also decrease the dosage received by PTV_63. Therefore, this case is rather challenging.

There are 1983 angles in the original data of this case and its size is more than 60GB. Since it may not be necessary to consider all the angles to obtain good results, we only select 180 of the angles to cover all the angles when the couch angle is zero. A summary of computational results is reported in Table 3. The results of RGAltMin are the average of 20 runs. Table 3 shows that our algorithms converge after fewer iterations with much less time, and reach a smaller value of the energy function. In particular, the randomized algorithm RGAltMin is significantly faster than GGAltMin. This demonstrates that introducing randomness into the alternating minimization framework is indeed helpful.

The dose distribution and their level-sets overlaying the outlined prostate and other or- 

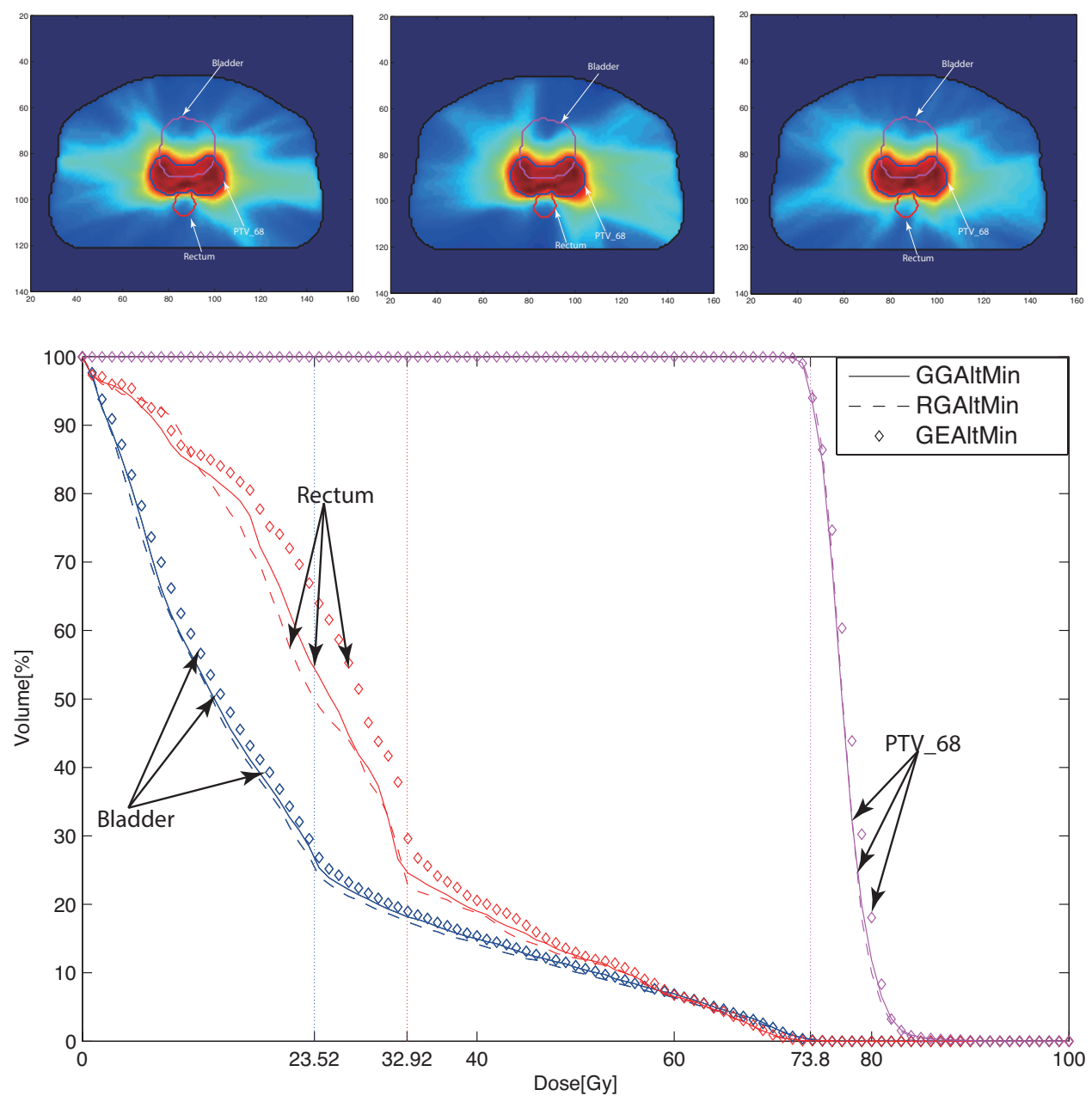

Fig. 5: Computational results on the prostate case. The three figures from left to right on the first row correspond to the dose distribution and their level-sets overlaying the outlined prostate and other organs on a chosen slice of a patient's scan obtained by GGAltMin, RGAltMin and GEAltMin, respectively. The second row shows the DVH curves for GGAltMin (solid lines), RGAltMin (dashed lines) and GEAltMin (lines with diamond markers).

gans on a chosen slice of a patient's scan as well as the DVH curves are plotted in the first of Figure 7. A summary of DVH is presented in Table 4 From these images and tables, the desired dosage distribution pattern are achieved by all algorithms. In particular, the GGAltMin curve shows that $90.75 \%$ of the PTV_63 and $91.97 \%$ of the PTV_70 are dosed at or above the required level, and $79.77 \%$ of the parotid and $86.50 \%$ of the spinal cord PRV are dosed below their safe limit in critical structures. The RGAltMin curve shows that $91.59 \%$ of the PTV_63 and $91.97 \%$ of the PTV_70 dosed at or above the required level, $83.02 \%$ of the parotid and $89.38 \%$ of the spinal cord PRV dosed below their safe limit. Hence, our algorithms can yield the desired characteristics of a good treatment plan with sufficient doses applied to target structures and safe doses applied to critical structures. The computational results of the 20 
runs of GEAltMin are further shown in Figure 6 using box plots. Even in the worst case, GEAltMin consumes a much shorter runtime and reaches a smaller energy E and less dose on the critical structures than the other two algorithms. Hence, the performance of GEAltMin is quite robust. Finally, the right side of Figure 8 shows some samples of the aperture shapes and beam intensities calculated by GGAltMin.

\begin{tabular}{cccc}
\hline algorithm & iteration & E & runtime \\
\hline GEAltMin & 300 & $5.28 e 8$ & $83 \mathrm{~m} 20 \mathrm{~s}$ \\
GGAltMin & 163 & $5.13 e 8$ & $38 \mathrm{~m} 11 \mathrm{~s}$ \\
RGAltMin & 71 & $4.55 e 8$ & $14 \mathrm{~m} 24 \mathrm{~s}$ \\
\hline
\end{tabular}

Table 3: A summary of computational results on the head-and-neck case.

\begin{tabular}{ccccc}
\hline algorithm & Parotid & Spinal cord PRV & PTV_63 & PTV_70 \\
\hline GEAltMin & $22.12 \%$ & $16.88 \%$ & $90.77 \%$ & $90.97 \%$ \\
GGAltMin & $20.23 \%$ & $13.50 \%$ & $90.75 \%$ & $91.97 \%$ \\
RGAltMin & $16.98 \%$ & $10.63 \%$ & $91.59 \%$ & $91.97 \%$ \\
\hline
\end{tabular}

Table 4: A summary of DVH on the head-and-neck case.
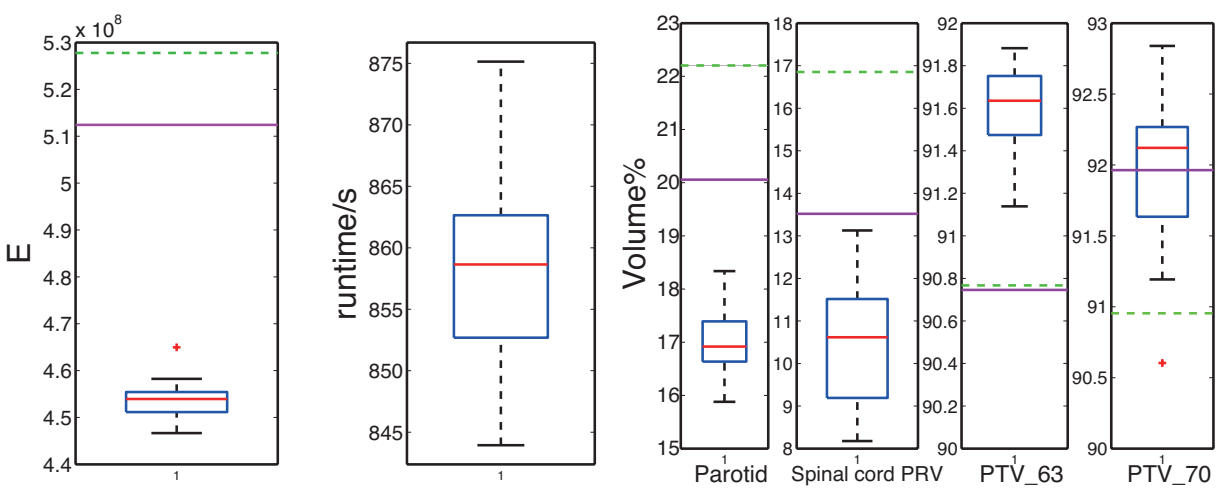

Fig. 6: Boxplots of the 20 runs of RGAltMin on the head-and-neck case. The figures from left to right are corresponding to energy, runtime and DVHs of Parotid, Spinal cord PRV, PTV_63 and PTV_70, respectively. The green dashed lines and the purple solid lines represent the corresponding values yielded by GEAltMin and GGAltMin, respectively. The runtimes of the GEAltMin and GGAltMin are not shown in the second figure since they are way larger than 875 seconds.

5. Conclusion. The optimization of VMAT treatment plans in cancer radiotherapy is an important problem in clinical application. The treatment plan optimization is complicated due to practical constraints imposed by the equipments involved. It requires to determine suitable aperture shapes and beam intensities in order to generate sufficient doses applied to target structures while minimizing doses applied to critical structures as much as possible. 

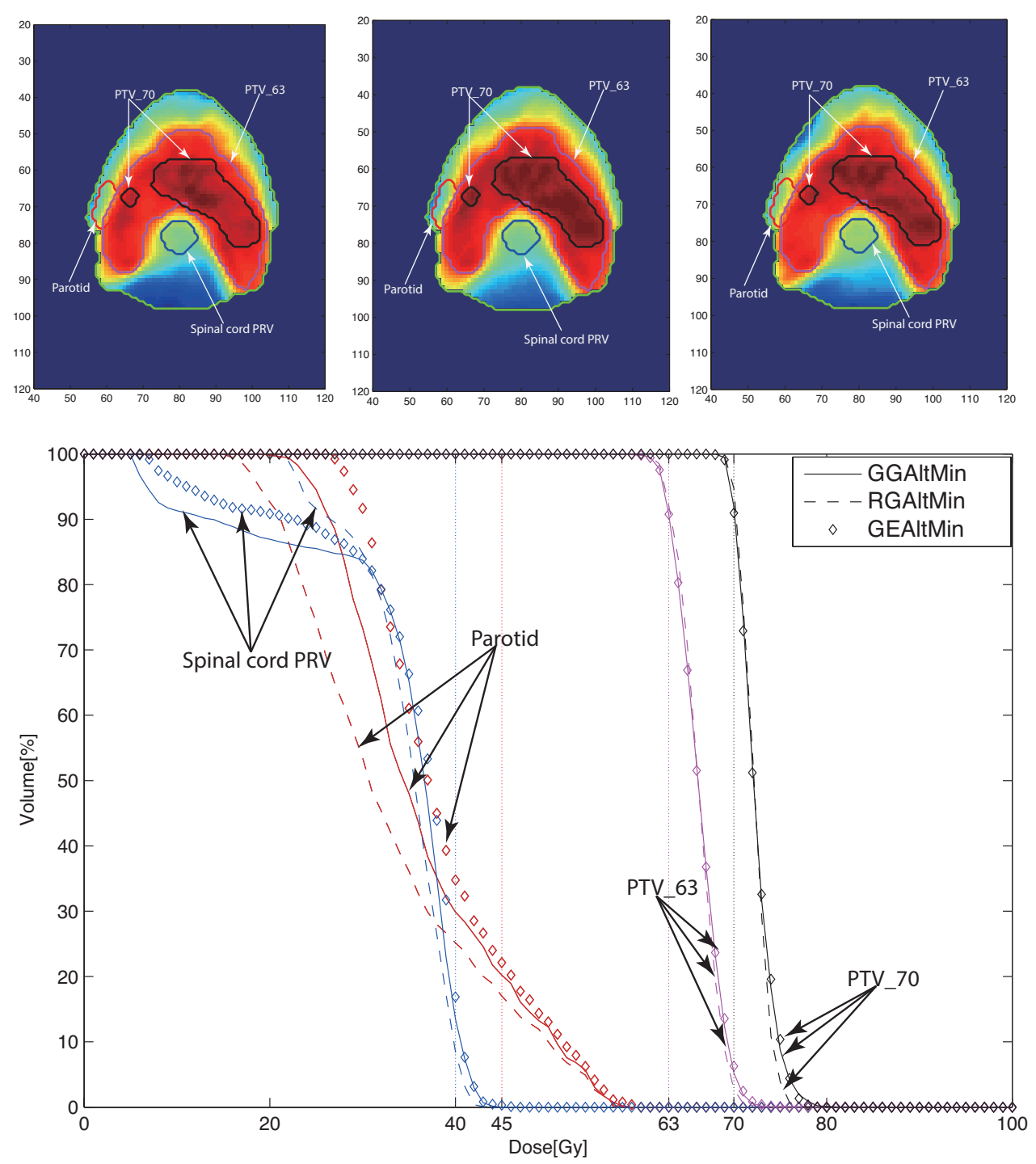

Fig. 7: Computational results on the head-and-neck case. The three figures from left to right on the first row correspond to the dose distribution and their level-sets overlaying the outlined prostate and other organs on a chosen slice of a patient's scan obtained by GGAltMin, RGAltMin and GEAltMin, respectively. The second row shows the DVH curves for GGAltMin (solid lines), RGAltMin (dashed lines) and GEAltMin (lines with diamond markers).

In this paper, we consider constraints on the bounds on the beam intensity and its rate of change, limit on the moving speed of the leaves of the multi-leaf collimator (MLC), and the direction-convexity of the MLC. We propose a mixed-integer nonlinear and nonconvex model in discrete setting where the aperture shapes are characterized by integer variables to conveniently describe the directional convexity properties. The model is solved by performing an alternating minimization with respect to the aperture shapes and the beam intensity, sepa- 

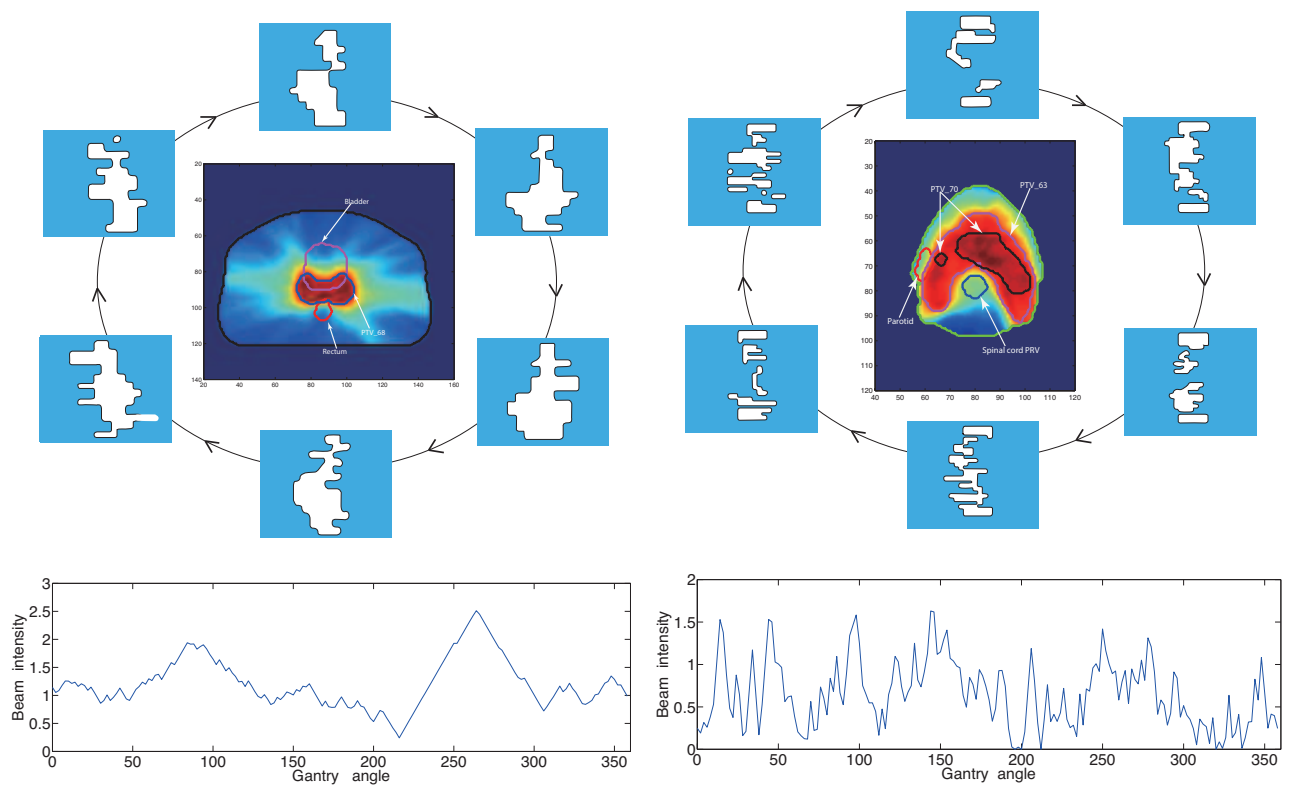

Fig. 8: A sample of the aperture shapes and beam intensities for the VMAT plans computed by GGAltMin in the case of prostate cancer (left) and head-and-neck cancer (right), respectively.

rately. The aperture shapes are computed using a greedy strategy which is further enhanced by random sampling. The beam intensities are computed by a standard gradient-projection method using nonmonotone line search. Since calculating the dose distribution and evaluating the energy function are computationally expensive due to the large number of voxels in the discrete setting, we propose an incremental randomized strategy which only computes a proportion of the voxels at a time. Comparing to the classical incremental algorithms, our selection of the voxels are based on probabilities defined by the importance of the voxels. Numerical simulations on the prostate and head-and-neck data sets confirm that our method is highly competitive to the state-of-the-art algorithms in terms of both computation efficiency and quality of treatment planning.

The performance of our algorithms can be further improved in several ways, such as speeding up convergence and improving accuracy, with the help of the recent techniques on mixed-integer programming and randomized optimization. Three particularly important topics for future investigations are (i) a comprehensive study of mathematical modelling of treatment planning in VMAT or other radiotherapy techniques, (ii) parallelizing the computation in the greedy approach and random sampling, and (iii) extensive numerical experiments on clinical data sets.

Acknowledgements. We would like to thank Dr. Xun Jia from Department of Radiation Oncology, University of Southwestern Medical Center for his valuable inputs on this project and the clinical data sets. We would also like to extend our gratitude to Dr. Li-Tien Cheng for sharing his source codes of the algorithms in [6]. 


\section{REFERENCES}

[1] Kerem Akartunali And Vicky MaK-HaU, Treatment planning optimization for volumetric-modulated arc therapy (vmat): Heuristic approaches.

[2] Guangying Zhu Bofei Sun, Jie Song And Leyuan Shi, A two-stage approach for vmat treatment plan optimization, International Conference on Automation Science, 6 (2013), pp. 455-460.

[3] Anders Brahme, J-E Roos, ANd Ingemar Lax, Solution of an integral equation encountered in rotation therapy, Physics in medicine and biology, 27 (1982), p. 1221.

[4] Chandra Burman, Chen-Shou Chui, Gerald Kutcher, Steven Leibel, Michael Zelefsky, Thomas LoSasso, Spiridon Spirou, Qiuwen Wu, Jie Yang, Jörg Stein, et al., Planning, delivery, and quality assurance of intensity-modulated radiotherapy using dynamic multileaf collimator: a strategy for large-scale implementation for the treatment of carcinoma of the prostate, International Journal of Radiation Oncology* Biology* Physics, 39 (1997), pp. 863-873.

[5] X. Y. Cedric AND G. TANG, Intensity-modulated arc therapy: Principles, technologies and clinical implementation, Phys. Med. Biol, 56 (2009), pp. 31-54.

[6] L.-T. Cheng, B. Dong, X. JiA C. Men, AND S. JiAng, Binary level-set shape optimization model and algorithm for volumetric modulated arc therapy in cancer radiotherapy, SIAM J. Sci. Comput, 35 (2012), pp. 1321-1340.

[7] David Craft, Mark Bangert, Troy Long, David Papp, and Jan Unkelbach, Shared data for intensity modulated radiationtherapy (imrt) optimization research: the cort dataset, Bio Med Central, 6 (2014).

[8] Palma D, Vollans E, James K, Nakano S, Moiseenko V, Shaffer R, McKen Zie M, Morris J, AND ОTто K, Volumetric modulated arc therapy for delivery of prostate radiotherapy: comparison with intensity-modulated radiotherapy and three dimensional conformal radiotherapy, Int.J. Radiat. Oncol. Biol. Phys, 74 (2008), pp. 996-1001.

[9] BARZILAI J AND BORWEIN J M, Two-point step size gradient methods, ima journal on numerical analysis, IMA journal of numerical analysis, 8 (1988), pp. 141-148.

[10] KARL OtTo, Volumetric modulated arc therapy: Imrt in a single gantry arc, Medical physics, 35 (2008), pp. 310-317.

[11] Fei Peng, Xun Jia, Xuejun Gu, Marina A Epelman, H Edwin Romeijn, and Steve B Jiang, A new column-generation-based algorithm for vmat treatment plan optimization, Phys. Med. Biol, 57 (2012), pp. 4569-4588.

[12] Verbakel W, Cuijpers J, Hoffmans D, Bieker M, Slotman B, and Senan S, Volumetric intensitymodulated arc therapy versus conventional imrt in head-and-neck cancer: a comparative planning and dosimetric study, Int.J. Radiat. Oncol. Biol. Phys, 74 (2009), pp. 252-259.

[13] C. Wang, S. Luan, G. Tang, D. Chen, M. Earl, And C. YU, Arc modulated radiation therapy: a single-arc form of intensity-modulated arc therapy, physics in medicine and biology, Phys. Med. Biol, 53 (2008), pp. 6291-6303.

[14] CEDRIC X YU, Intensity-modulated arc therapy with dynamic multileaf collimation: an alternative to tomotherapy, Physics in medicine and biology, 40 (1995), p. 1435.

[15] Zhanga, HONGCHAO, HAGER, AND WILliam W., A nonmonotone line search technique and its application to unconstrained optimization, SIAM J. Optim., 14 (2004), pp. 1043-1056. 